\title{
Dynamical instability of symmetric vortices
}

Luís Almeida and Yan Guo

Abstract. Using the Maxwell-Higgs model, we prove that linearly unstable symmetric vortices in the Ginzburg-Landau theory are dynamically unstable in the $H^{1}$ norm (which is the natural norm for the problem).

In this work we study the dynamic instability of the radial solutions of the Ginzburg-Landau equations in $\mathbb{R}^{2}$,

$$
\left\{\begin{array}{l}
\operatorname{curl}^{2} A+\frac{i}{2}(\bar{\phi} D \phi-\phi \overline{D \phi})=0 \\
-D^{2} \phi+\frac{\lambda}{2}\left(|\phi|^{2}-1\right) \phi=0
\end{array}\right.
$$

where $\phi: \mathbb{R}^{2} \longrightarrow \mathbb{C}$ is the Higgs field, or condensed wave function ( $|\phi|^{2}$ is proportional to the local density of Cooper pairs), and $A$ is the gauge potential 1-form (it can also be seen as the vector potential of the magnetic field). The covariant derivative is $D \phi=\nabla \phi-i A \phi$, with $i=\sqrt{-1}$. The electric field is absent in the stationary model, and $H=\operatorname{curl} A$ is the magnetic field. The dimensionless coupling constant $\lambda$ is positive, $\lambda<1$ corresponding to superconductors of type I and $\lambda>1$ to those of type II.

Solutions of (1) are critical points of the Helmholtz free energy associated to the Ginzburg-Landau model, which we may write as

$$
\mathcal{E}=\int_{\mathbb{R}^{2}}\left(\frac{1}{2}|\operatorname{curl} A|^{2}+\frac{1}{2}|D \phi|^{2}+\frac{\lambda}{8}\left(|\phi|^{2}-1\right)^{2}\right)
$$


There is a vortex number (charge) associated with every finite energy solution of (1). It can be defined as

$$
n:=\frac{1}{2 \pi} \int_{\mathbb{R}^{2}} H=\frac{1}{2 \pi} \lim _{N \rightarrow \infty} \int_{|x|=N} A d x .
$$

This number, which is always an integer, has a topological meaning it is the winding number of the Higgs field $\phi$ (see, for instance, [7]).

In the early seventies, Nielson and Olesen ([8]) interpreted the finite energy solutions of (1) as string-like field configurations and, soon after, a family of topologically non-trivial solutions (one for every integer value of the topological degree $n$ and positive real value of the parameter $\lambda$ ) was constructed mathematically by Berger and Chen ([2]) and Plohr $([9])$. In polar coordinates $(r, \theta) \in \mathbb{R}^{2}$ these radial solutions $(a, \eta)$ are of the form

$$
a(r, \theta)=a_{1} d x_{1}+a_{2} d x_{2}=n S(r) d \theta, \quad \eta(r, \theta)=R(r) e^{i n \theta},
$$

where $n \in \mathbb{Z}$ and $\lambda>0$ are arbitrary. Here $r=\sqrt{\left(x^{1}\right)^{2}+\left(x^{2}\right)^{2}}$ and $\theta=\tan ^{-1}\left(x^{2} / x^{1}\right)$.

For studying the dynamics, one should consider the action on the Minkowski space-time $\mathbb{R}^{1+2}$ (we add the time coordinate, $t$, which will also be denoted by $x^{0}$ ), with a metric $g^{\mu \nu}, \mu, \nu=0,1,2$, with signature $(-,+,+)$. The action is then given by (see $[7,1.9 . a)$ and $b)])$

$$
\begin{aligned}
\mathcal{A} & =\frac{1}{2} \int_{\mathbb{R}^{1+2}}\left(\left|F_{12}\right|^{2}-\left|D_{0} \phi\right|^{2}+\sum_{j=1}^{2}\left(\left|D_{j} \phi\right|^{2}-\left|F_{0 j}\right|^{2}\right)+\frac{\lambda}{4}\left(|\phi|^{2}-1\right)^{2}\right) \\
& \text { (4) } \\
& =\frac{1}{2} \int_{\mathbb{R}^{1+2}}\left(g^{i j} g^{k l} F_{i k} F_{j l}+g^{i j} D_{i} \phi \overline{D_{j} \phi}+\frac{\lambda}{4}\left(|\phi|^{2}-1\right)^{2}\right),
\end{aligned}
$$

where, in the last expression we used the Einstein convention for summing over repeated indices (we will continue to do so below). Here, $A$ is now the electro-magnetic potential - it has also an electric potential component $A_{0}$. We denote partial derivatives by $\partial_{\mu}=\partial_{x^{\mu}}$, for $\mu=0,1,2$. Then, since $F_{\mu \nu}=\partial_{\mu} A_{\nu}-\partial_{\nu} A_{\mu}$, for $0 \leq \mu, \nu \leq 2$, the electric field is given by $F_{0 j}, j=1,2$, and $-F_{12}$ is the magnetic field. We denoted covariant derivatives with respect to space or time variables by $D_{\mu}=\partial_{\mu}-i A_{\mu}$. As usual, we will raise and lower indices by using the metric $g$. For instance, $D^{i}:=g^{i j} D_{j}$, and thus $D^{0}=-D_{0}$, while $D^{1}=D_{1}$. 
Dynamical stability is then investigated using the Maxwell-Higgs system, which can be written as

$$
\left\{\begin{array}{l}
\partial^{\mu} F_{\mu \nu}=-j_{\nu}, \\
D_{\mu} D^{\mu} \phi+\frac{\lambda}{2}\left(|\phi|^{2}-1\right) \phi=0 .
\end{array}\right.
$$

The charge and current densities are given by

$$
j_{\nu}=\operatorname{Im}\left(\phi \overline{D_{\nu} \phi}\right)=-\frac{i}{2}\left(\phi \overline{D_{\nu} \phi}-\bar{\phi} D_{\nu} \phi\right),
$$

for $\mu=0,1,2$, and the conserved energy is

$$
\frac{1}{2} \int_{\mathbb{R}^{2}}\left(\left|F_{\mu \nu}\right|^{2}+\left|D_{\mu} \phi\right|^{2}+\frac{\lambda}{4}\left(|\phi|^{2}-1\right)^{2}\right) d x^{1} d x^{2} .
$$

The Maxwell-Higgs model is invariant under a gauge transformation

$$
\left\{\begin{array}{l}
A_{\mu} \longrightarrow A_{\mu}+\partial_{\mu} \chi \\
\phi \rightarrow e^{i \chi} \phi
\end{array}\right.
$$

We will work under the temporal gauge condition $A_{0} \equiv 0$, and thus we just need to consider the variations of the spatial components of $A$ (we will be back to working with a 2 -dimensional $A$, with real components $A_{1}$ and $A_{2}$, and a $\mathbb{C} \simeq \mathbb{R}^{2}$ valued Higgs field $\left.\phi\right)$. Let $v=(W, \psi)^{T} \in \mathbb{R}^{4}$ be a perturbation of the radially symmetric vortex $(a, \eta)$, where $W=$ $A-a$ and $\psi=\phi-\eta$. The full nonlinear Maxwell-Higgs system, in terms of $v$, can be written as

$$
\left\{\begin{array}{l}
\partial_{t}\left(\partial_{1} W_{1}+\partial_{2} W_{2}\right)-\frac{i}{2} \partial_{t}(\eta \bar{\psi}-\bar{\eta} \psi)=\frac{i}{2}\left(\psi \partial_{t} \bar{\psi}-\bar{\psi} \partial_{t} \psi\right), \\
\frac{d^{2} v}{d t^{2}}+\mathcal{E}^{\prime \prime}{ }_{(a, \eta)} v=N(v)
\end{array}\right.
$$

Here, $\mathcal{E}^{\prime \prime}{ }_{(a, \eta)}$ denotes the second order variation of (2) around the vortices $(a, \eta)$, and the nonlinear term $N(v)$ is equal to

$$
\left(\begin{array}{c}
\frac{i}{2}\left(\psi \partial_{k} \bar{\psi}-\bar{\psi} \partial_{k} \psi\right)-W_{k}|\psi|^{2}-W_{k}(\eta \bar{\psi}+\bar{\eta} \psi)-a_{k}|\psi|^{2} \\
-i W_{j} \partial_{j} \psi-i \partial_{j}\left(W_{j} \psi\right)-W_{j}^{2} \psi-2 a_{j} W_{j} \psi \\
-\eta W_{j}^{2}-\frac{\lambda}{2}\left(|\psi|^{2}(\psi+2 \eta)+\psi^{2} \bar{\eta}\right)
\end{array}\right)
$$


where $1 \leq k \leq 2$, and we have an implicit sum over $1 \leq j \leq 2$.

Ever since the construction of these stationary radially symmetric vortices, their stability against initial perturbations with the same charge has been an interesting problem for both mathematicians and physicists. In a classical paper of [3], the question of stability was addressed by numerical and formal analysis. The study of the linear operator $\mathcal{E}^{\prime \prime}{ }_{(a, \eta)}$ indicates that for $\lambda \leq 1$ and all charges $n$ the vortices are linearly stable. On the other hand, for $\lambda>1$ and $|n| \geq 2$, the vortices are linearly unstable. Unlike in the finite dimensional dynamical system, the passage form linear growing modes to a genuine nonlinear instability in an infinite dimensional partial differential equation is quite delicate. This is due to the possible presence of the continuous spectrum for the linearized operator and to severe high order perturbations arising from the nonlinearity.

In previous works ([4] and [1]), the dynamical instability of vortices with large coupling constant $\lambda$ was proven in the norm

$$
\|f\|_{X}=\|f\|_{H^{1}\left(\mathbb{R}^{2}\right)}+\|f\|_{\infty} .
$$

The $\|\cdot\|_{\infty}$ was needed to control the $H^{2}$ growth estimate.

In this work, we improve the passage from linear instability to nonlinear dynamical instability in the more natural $H^{1}$ norm by a refined bootstrap argument. Let the initial perturbation be of the order $\delta$. Within a time-interval of the order of $|\ln \delta|$ we can estimate the $H^{2}$ norm of the perturbation only by its $H^{1}$ norm (without any extra assumptions on its $L^{\infty}$ norm). A similar argument has also been used in [5].

In fact, in Theorem 1, we show that if the linear operator $\mathcal{E}^{\prime \prime}(a, \eta)$ has a negative direction, then the vortex is dynamically unstable in $H^{1}$ norm.

For given positive constants $\Omega$ and $\varepsilon_{0}$, and for any small parameter $\delta>0$, we define the associated escape time $T^{\delta}$ by

$$
\delta e^{\Omega T^{\delta}}=\varepsilon_{0} .
$$

For a fixed appropriately chosen $\varepsilon_{0}$, as $\delta \longrightarrow 0$ the dynamical instability will occur within $0 \leq t \leq T^{\delta}$.

Lemma 1. Let $v(t)$ be a solution of the full Maxwell-Higgs system (6). Assume

$$
\begin{gathered}
\|v(0)\|_{H^{2}}+\left\|v_{t}(0)\right\|_{H^{1}} \leq C_{0} \delta, \\
\|v(t)\|_{H^{1}}+\left\|v_{t}(t)\right\|_{2} \leq C_{0} e^{\Omega t} \delta,
\end{gathered}
$$


for $0 \leq t \leq T$, where $\Omega>0$ and $C_{0}$ is independent of $t$. Then, there exist $C_{1}, \varepsilon_{0}>0$ such that if $0 \leq t \leq \min \left\{T, T^{\delta}\right\}$ then

$$
\|v(t)\|_{H^{2}}+\left\|v_{t}(t)\right\|_{H^{1}} \leq C_{1} \delta e^{\Omega t} \leq C_{1} \varepsilon_{0},
$$

where $T^{\delta}$ is defined in (7).

Proof. We shall estimate $\|v\|_{H^{2}}$ in terms of $\|v\|_{H^{1}}$ by energy type estimates. Taking one spatial derivative $\partial_{l}=\partial_{x^{l}}$ through both equations in (6) we obtain

$$
\left\{\begin{aligned}
\partial_{t}\left(\partial_{1} \partial_{l} W_{1}+\partial_{2} \partial_{l} W_{2}\right)-\frac{i}{2} \partial_{t}\left(\eta \partial_{l} \bar{\psi}-\bar{\eta} \partial_{l} \psi\right) \\
=\frac{i}{2} \partial_{t}\left(\partial_{l} \eta \bar{\psi}-\partial_{l} \bar{\eta} \psi\right)+\frac{i}{2} \partial_{l}\left(\psi \partial_{t} \bar{\psi}-\bar{\psi} \partial_{t} \psi\right) \\
\frac{d^{2}}{d t^{2}}\left(\partial_{l} v\right)-L\left(\partial_{l} v\right)=L_{1}(v)+\partial_{l} N(v)
\end{aligned}\right.
$$

Here $L_{1}(v)$ is

$$
\left(\begin{array}{c}
\frac{i}{2}\left(\partial_{l} \eta \partial_{k} \bar{\psi}+\psi \partial_{k l} \bar{\eta}-\partial_{l} \bar{\eta} \partial_{k} \psi-\bar{\psi} \partial_{k l} \eta\right) \\
-\partial_{l} a_{k}(\eta \bar{\psi}+\bar{\eta} \psi)-a_{k}\left(\partial_{l} \eta \bar{\psi}+\partial_{l} \bar{\eta} \psi\right)-W_{k} \partial_{l}|\eta|^{2} \\
-2 i \partial_{l} a_{j} \partial_{j} \psi-2 i W_{j} \partial_{j l} \eta-i \partial_{j} W_{j} \partial_{l} \eta-\partial_{l}\left(|a|^{2}+\lambda|\eta|^{2}\right) \psi \\
-2 W_{j} \partial_{l}\left(a_{j} \eta\right)-\frac{\lambda}{2} \partial_{l}\left(\eta^{2}\right) \bar{\psi}
\end{array}\right)
$$

Define $y(t):=\|v(t)\|_{H^{2}}^{2}+\left\|v_{t}(t)\right\|_{H^{1}}^{2}$. Using estimate (3.18) in [4] with sufficiently small $\varepsilon$, we have that

$$
\begin{aligned}
y(t) \leq & \left(C\left(\|v\|_{X}+\|v\|_{X}^{2}\right)+\frac{3 \Omega}{16}\right) \int_{0}^{t} y(\tau) d \tau \\
& +C_{\Omega} \int_{0}^{t}\left(\|v\|_{H^{1}}^{2}+\left\|v_{t}\right\|_{2}^{2}\right) d \tau \\
& +C\|v(t)\|_{H^{1}}^{2}+C\left(\|v(0)\|_{H^{2}}^{2}+\left\|v_{t}(0)\right\|_{H^{1}}^{2}\right) .
\end{aligned}
$$

We notice that due to typographical errors, the square was omitted in (3.18) for both $\|v(t)\|_{H^{1}}$ and the initial data $\|v(0)\|_{H^{2}}+\left\|v_{t}(0)\right\|_{H^{1}}$. 
Since $\|v\|_{X} \leq C\|v\|_{H^{2}}$

$$
\begin{aligned}
y(t) \leq & \left(\bar{C}\left(\|v\|_{H^{2}}+\|v\|_{H^{2}}^{2}\right)+\frac{3 \Omega}{16}\right) \int_{0}^{t} y(\tau) d \tau \\
& +C_{\Omega} \int_{0}^{t}\left(\|v\|_{H^{1}}^{2}+\left\|v_{t}\right\|_{2}^{2}\right) d \tau \\
& +C\|v(t)\|_{H^{1}}^{2}+C\left(\|v(0)\|_{H^{2}}^{2}+\left\|v_{t}(0)\right\|_{H^{1}}^{2}\right) .
\end{aligned}
$$

We now define

$$
\begin{aligned}
& T^{*}=\sup \{t: \text { for all } s \in[0, t], \\
& \left.\qquad\|v(s)\|_{H^{2}}+\left\|v_{t}(s)\right\|_{H^{1}} \leq \min \left\{\frac{\Omega}{8 \bar{C}}, 1\right\}\right\} .
\end{aligned}
$$

For $0 \leq t \leq \min \left\{T, T^{*}\right\}$, since $\|v(t)\|_{H^{2}} \leq 1$, we have

$$
\bar{C}\left(\|v\|_{H^{2}}+\|v\|_{H^{2}}^{2}\right) \leq \frac{\Omega}{4} .
$$

Moreover, from (9),

$$
\begin{aligned}
C_{\Omega} \int_{0}^{t}\left(\|v\|_{H^{1}}^{2}+\left\|v_{t}\right\|_{2}^{2}\right) d \tau+C\|v(t)\|_{H^{1}}^{2} \\
\leq C_{\Omega} \int_{0}^{t}\left(C_{0} \delta e^{\Omega \tau}\right)^{2} d \tau+C \delta^{2} e^{2 \Omega t} \\
\leq C \delta^{2} e^{2 \Omega t}
\end{aligned}
$$

Therefore, using (8), we obtain from (13)

$$
y(t) \leq \frac{\Omega}{2} \int_{0}^{t} y(\tau) d \tau+C \delta^{2} e^{2 \Omega t}
$$

Now, proceeding as in the proof of the Gronwall inequality, we deduce that

$$
\left(e^{-(\Omega / 2) t} \int_{0}^{t} y(\tau) d \tau\right)^{\prime} \leq C \delta^{2} e^{2 \Omega t-(1 / 2) \Omega t}=C \delta^{2} e^{(3 / 2) \Omega t}
$$

Integrating over $t$, we obtain

$$
e^{-(\Omega / 2) t} \int_{0}^{t} y(\tau) d \tau \leq C \delta^{2} \int_{0}^{t} e^{(3 / 2) \Omega s} d s=C \delta^{2} e^{(3 / 2) \Omega t}
$$


Therefore,

$$
\int_{0}^{t} y(\tau) d \tau \leq C \delta^{2} e^{2 \Omega t}
$$

And plugging this into (15) yields

$$
\|v(t)\|_{H^{2}}+\left\|v_{t}(t)\right\|_{H^{1}} \leq C_{1} \delta e^{\Omega t},
$$

for $0 \leq t \leq \min \left\{T, T^{*}\right\}$, where $C_{1}$ is some fixed constant which depends on $\Omega$ and $C_{0}$, but is independent of $\delta$.

We now define $T^{\delta}$ as in (7), choosing $\varepsilon_{0}$ such that

$$
C_{1} \varepsilon_{0}<\min \left\{\frac{\Omega}{8 \bar{C}}, 1\right\}
$$

Then, if $T^{\delta} \leq \min \left\{T, T^{*}\right\}$, clearly the lemma follows. On the other hand, if $T^{\delta}>\min \left\{T, T^{*}\right\}$, we claim that $T \leq T^{*}$. It thus follows that $\min \left\{T, T^{*}\right\}=T$ and, once more, the lemma follows easily.

To prove $T \leq T^{*}$, we argue by contradiction. If not, we would have $T>T^{*}$ and therefore $\min \left\{T, T^{*}\right\}=T^{*}$. Letting $t=T^{*}$ in (16) would yield

$$
\left\|v\left(T^{*}\right)\right\|_{H^{2}}+\left\|v_{t}\left(T^{*}\right)\right\|_{H^{1}} \leq C_{1} \delta e^{\Omega T^{*}}<C_{1} \delta e^{\Omega T^{\delta}}=C_{1} \varepsilon_{0}
$$

by the definition of $T^{\delta}$. However, this is impossible by the choice (17) since it would contradict the definition of $T^{*}$ in (14).

Now, we may prove our main result.

Theorem 1. Let $(a, \eta)$ be a vortex such that

$$
\left\langle\mathcal{E}^{\prime \prime}{ }_{(a, \eta)}\left(v_{1}\right), v_{1}\right\rangle<0,
$$

for some $v_{1} \in H^{1}\left(\mathbb{R}^{2}\right)$. Then, there exist constants $\varepsilon_{0}>0, C>0$, so that for any small $\delta>0$ there exists a family of solutions $v^{\delta}(t)$ of the Maxwell-Higgs system (6) such that the vortex number of $W^{\delta}(0)$ is zero, and

$$
\left\|v^{\delta}(0)\right\|_{H^{2}}+\left\|v_{t}^{\delta}(0)\right\|_{H^{1}} \leq C \delta
$$

but

$$
\sup _{\{0 \leq t \leq C|\ln \delta|\}}\left\|v^{\delta}(t)\right\|_{H^{1}}+\left\|v_{t}^{\delta}(t)\right\|_{L^{2}} \geq \frac{\varepsilon_{0}}{2}
$$


Proof. By [4, Theorem 1.3], there exists a dominant growing mode $v_{0} e^{\omega t}$ of the linearized Maxwell-Higgs system with $\omega>0$ and $v_{0} \in$ $H^{2}\left(\mathbb{R}^{2}\right)$. We normalize $v_{0}$ such that

$$
\left\|v_{0}\right\|_{H^{1}}+\left\|\omega v_{0}\right\|_{L^{2}}=1 \text {. }
$$

Moreover, we assume that

$$
\left\|v_{0}\right\|_{H^{2}}+\left\|\omega v_{0}\right\|_{H^{1}}=r<\infty .
$$

Now we solve the Maxwell-Higgs system with a family of initial data $\left.v\right|_{t=0}=\delta v_{0}$ and $\left.v_{t}\right|_{t=0}=\delta \omega v_{0}$. Notice that the vortex number (charge) of $a+W$ is the same as that of $a$. We denote the corresponding $H^{2}$ solutions by $v^{\delta}(t)$. They can be written as

$$
v^{\delta}(t)=\delta e^{\omega t} v_{0}+\int_{0}^{t} \mathcal{L}(t-\tau) \mathcal{N}\left(v^{\delta}\right) d \tau,
$$

where $\mathcal{L}$ is the solution operator for the linearized Maxwell-Higgs system, and

$$
\mathcal{N}\left(v^{\delta}\right)=\left(\frac{i}{2}\left(\psi^{\delta} \partial_{t} \bar{\psi}^{\delta}-\bar{\psi}^{\delta} \partial_{t} \psi^{\delta}\right), N\left(v^{\delta}\right)\right) .
$$

Let $\omega \leq \Omega<2 \omega$, and

$$
T=\sup \{s: \text { for all } t \in[0, s],
$$

$$
\left.\left\|v^{\delta}(t)-\delta v_{0} e^{\omega t}\right\|_{H^{1}}+\left\|v_{t}^{\delta}(t)-\omega \delta v_{0} e^{\omega t}\right\|_{2} \leq \frac{1}{2} \delta e^{\omega t}\right\}
$$

Using the triangle inequality, we see that for $0 \leq t \leq T$,

$$
\begin{aligned}
\left\|v^{\delta}(t)\right\|_{H^{1}}+\left\|v_{t}^{\delta}(t)\right\|_{2} \leq & \left\|\delta v_{0} e^{\omega t}\right\|_{H^{1}}+\left\|\omega \delta v_{0} e^{\omega t}\right\|_{2} \\
& +\left\|v^{\delta}(t)-\delta v_{0} e^{\omega t}\right\|_{H^{1}}+\left\|v_{t}^{\delta}(t)-\omega \delta v_{0} e^{\omega t}\right\|_{2} \\
& \leq \frac{3}{2} \delta e^{\omega t}\left(\left\|v_{0}\right\|_{H^{1}}+\left\|\omega v_{0}\right\|_{2}\right) \\
& =\frac{3}{2} e^{\omega t} \delta .
\end{aligned}
$$

For any $\varepsilon>0$, from the sharp linear estimate for $\mathcal{L}$ as in $[4$, Theorems 1.3 and 1.5], we know that the solutions of the linearized GinzburgLandau equation grow no faster than $e^{(\omega+\varepsilon) t}$. By Lemma 1 with $\Omega=\omega$, 
$C_{0}=\max \{3 / 2, r\}$, there exist constants $C_{1}, \varepsilon_{0}>0$ such that for $0 \leq t \leq \min \left\{T, T^{\delta}\right\}$,

$$
\left\|v^{\delta}(t)\right\|_{H^{2}}+\left\|v_{t}^{\delta}(t)\right\|_{2} \leq C_{1} \delta e^{\omega t} \leq C_{1} \varepsilon_{0} .
$$

From the Sobolev embedding,

$$
\left\|v^{\delta}(t)\right\|_{\infty} \leq C\left\|v^{\delta}(t)\right\|_{H^{2}} \leq C \delta e^{\omega t} \leq C C_{1} \varepsilon_{0},
$$

for $0 \leq t \leq \min \left\{T, T^{\delta}\right\}$, where $\delta e^{\omega T^{\delta}}=\varepsilon_{0}$. From the linearized estimate (20) together with the linear estimate in [4, Theorem 1.5], for $0 \leq t \leq \min \left\{T, T^{\delta}\right\}$,

$$
\begin{aligned}
& \left\|v^{\delta}(t)-\delta e^{\omega t} v_{0}\right\|_{H^{1}}+\left\|v_{t}^{\delta}(t)-\delta \omega e^{\omega t} v_{0}\right\|_{2} \\
& \leq C \int_{0}^{t} e^{(3 / 2) \omega(t-\tau)}\left(\left\|\frac{i}{2}\left(\psi^{\delta} \partial_{t} \bar{\psi}^{\delta}-\bar{\psi}^{\delta} \partial_{t} \psi^{\delta}\right)\right\|_{2}+\left\|N\left(v^{\delta}\right)\right\|_{2}\right) d \tau \\
& \leq C \int_{0}^{t} e^{(3 / 2) \omega(t-\tau)}\left(\left\|v^{\delta}(\tau)\right\|_{\infty}\left\|v^{\delta}(\tau)\right\|_{H^{1}}+\left\|v^{\delta}(\tau)\right\|_{\infty}^{2}\left\|v^{\delta}(\tau)\right\|_{2}\right) d \tau \\
& \leq C \int_{0}^{t} e^{(3 / 2) \omega(t-\tau)}\left\|v^{\delta}(\tau)\right\|_{\infty}\left\|v^{\delta}(\tau)\right\|_{H^{1}} d \tau
\end{aligned}
$$

$$
\begin{aligned}
& \leq C \int_{0}^{t} e^{(3 / 2) \omega(t-\tau)}\left(\delta e^{\omega \tau}\right)\left(\delta e^{\omega \tau}\right) d \tau \\
& =C \delta^{2} e^{(3 / 2) \omega t} \int_{0}^{t} e^{(1 / 2) \omega \tau} d \tau \\
& \leq C_{2}\left(\delta e^{\omega t}\right)^{2}
\end{aligned}
$$

where $C_{2}$ is a constant.

Notice that both Lemma 1 and (23) remain valid with the same constants $C_{1}$ and $C_{2}$, respectively, for all smaller $\varepsilon_{0}$, as long as we take the corresponding $T^{\delta}$ in (7). In particular, if necessary we can fix $\varepsilon_{0}$ sufficiently small so that

$$
C_{2} \varepsilon_{0} \leq \frac{1}{2}
$$

Now, clearly $T^{\delta} \leq T$. Otherwise, we would have $T<T^{\delta}$, and from (19), (23), (22) and (24) it would follow that

$$
\left\|v^{\delta}(T)\right\|_{H^{1}}+\left\|v_{t}^{\delta}(T)\right\|_{2} \leq \delta e^{\omega T}+\left(C_{2} \delta e^{\omega T^{\delta}}\right) \delta e^{\omega T}<\frac{3}{2} \delta e^{\omega T}
$$


which would contradict the definition of $T$ in (21).

Once we know $T^{\delta} \leq T$, using (7), (19) and (23) again, we see that

$$
\begin{aligned}
\left\|v^{\delta}\left(T^{\delta}\right)\right\|_{H^{1}}+\left\|v_{t}^{\delta}\left(T^{\delta}\right)\right\|_{2} \geq & \delta e^{\omega T^{\delta}}\left(\left\|v_{0}\right\|_{H^{1}}+\left\|\omega v_{0}\right\|_{2}\right) \\
& -\left\|v^{\delta}\left(T^{\delta}\right)-\delta v_{0} e^{\omega T^{\delta}}\right\|_{H^{1}} \\
& -\left\|v_{t}^{\delta}\left(T^{\delta}\right)-\omega \delta v_{0} e^{\omega T^{\delta}}\right\|_{2} \\
\geq & \delta e^{\omega T^{\delta}}-C_{2} \varepsilon_{0} \delta e^{\omega T^{\delta}} \\
\geq & \frac{\varepsilon_{0}}{2} \\
& >0
\end{aligned}
$$

and the proof is complete.

REMARK. In [4] and [1], we had already proved that when $|n|>1$, such negative directions exist for large $\lambda$. Very recently, Gustafson and Sigal ([6]) proved that for all $\lambda>1$ and $|n|>1$, there exists a $v_{1}$ such that $\left\langle\mathcal{E}^{\prime \prime}{ }_{(a, \eta)}\left(v_{1}\right), v_{1}\right\rangle<0$. Thus, we obtain as an easy corollary of Theorem 1, the dynamic instability of symmetric vortices when $|n|>1$ and $\lambda>1$.

Corollary 1. For all $\lambda>1$, radially symmetric solutions $(a, \eta)$ of Ginzburg-Landau equations (i.e. solutions of (1) of the form (3)) with $|n|>1$, are dynamically unstable in $H^{1}$ norm, in the sense of Theorem 1 .

Acknowledgement. We thank the referee for helpful comments.

\section{References.}

[1] Almeida, L., Bethuel, F., Guo, Y., A remark on the instability of symmetric vortices with large coupling constant. Comm. Pure Appl. Math. L (1997), 1295-1300.

[2] Berger M. S., Chen Y. Y., Symmetric vortices for the Ginzberg-Landau equations of superconductivity and the nonlinear desingularization phenomenon. J. Funct. Anal. 82 (1989), 259-295. 
[3] Bogomol'nyi, E. B., The stability of classical solutions. Sov. J. Nucl. Phys. 24 (1976), 449-454.

[4] Guo Y., Instability of the symmetric vortices with large charge and coupling constant. Comm. Pure Appl. Math. 49 (1996), 1051-1080.

[5] Guo, Y., Strauss, W., Relativistic unstable periodic BGK waves. Comm. Pure Appl. Math. 8 (1999), 87-122.

[6] Gustafson, S., Sigal, I. M., The stability of magnetic vortices. Comm. Math. Phys. 212 (2000), 257-275.

[7] Jaffe, A., Taubes, C. H., Vortices and Monopoles. Birkhauser, 1980.

[8] Nielson, H. B., Olesen, P., Vortex-line models for dual strings. Nucl. Phys. B 61 (1973), 45-61.

[9] Plohr, B. J., Thesis. Princeton University, 1980.

Recibido: 17 de septiembre de 1.999

Revisado: 16 de febrero de 2.000

Luís Almeida

Laboratoire J. A. Dieudonne

UMR CNRS 6621

Universite de Nice Sophia-Antipolis

Parc Valrose

06108 Nice Cédex 2, FRANCE

luis@math.unice.fr

and

Yan Guo

Lefschetz Center for Dynamical Systems

Division of Applied Mathematics

Brown University

Providence, RI 02912, U.S.A.

guoy@cfm. brown.edu 